http://dx.doi.org/10.21707/gs.v11.n04a07

\title{
EFEITO DOS ESTRESSES HÍDRICO, SALINO E TÉRMICO NA GERMINAÇÃO DE SEMENTES DE HaNDROANTHUS spongiosus (RIZZINI) S. Grose (Bignoniaceae)
}

\author{
Jéssica Viviane Amorim Ferreira ${ }^{1 *}$, Marcos Vinicius Meiado ${ }^{2}$, José Alves de Siqueira Filho $^{1}$
}

${ }^{1}$ Colegiado Acadêmico de Ciências Biológicas, Campus de Ciências Agrárias, Universidade Federal do Vale do São Francisco. BR 407, Km 12, Lote 543, Projeto de Irrigação Nilo Coelho C1, Petrolina, Pernambuco, Brasil. CEP: 56300-990.

${ }^{2}$ Laboratório de Fisiologia de Sementes, Departamento de Biociências, Universidade Federal de Sergipe. Av. Vereador Olimpio Grande, s/n, Bloco D, Campus Professor Alberto Carvalho, Bairro Porto, Itabaiana, Sergipe, Brasil. CEP: 49510-200.

* Autor para correspondência: jessicaviviane.f@gmail.com

Recebido em 22 de novembro de 2016. Aceito em 06 de maio de 2017. Publicado em 30 de dezembro de 2017.

Resumo - O objetivo deste estudo foi avaliar a morfometria do fruto e as respostas germinativas de sementes de Handroanthus spongiosus (Rizzini) S. Grose (Bignoniaceae) submetidas a fatores ambientais, assim como contribuir para o conhecimento da ecofisiologia dessa espécie ameaçada de extinção. As sementes foram coletadas no município de Casa Nova, localizado Norte do Estado da Bahia. Foi determinada a temperatura ideal para germinação das sementes, além de avaliar o efeito da temperatura e dos estresses hídrico e salino na germinação das sementes. Também foram avaliados o peso, o comprimento e a quantidade de sementes em cada fruto da espécie. Foi observado que não há uma uniformidade no tamanho e na quantidade de sementes presente nos frutos. A temperatura ideal para a germinação das sementes foi $25^{\circ} \mathrm{C}$ e a espécie se mostrou tolerante a faixa de temperatura de 15 a $35^{\circ} \mathrm{C}$. Observou-se, ainda, que $H$. spongiosus não se mostrou tolerante aos estresses hídrico e salino, o que pode estar relacionado à distribuição restrita da espécie em algumas regiões da Caatinga.

Palavras-chave: CaAtinga; Cascudo; Disponibilidade Hídrica; Salinidade; Temperatura.

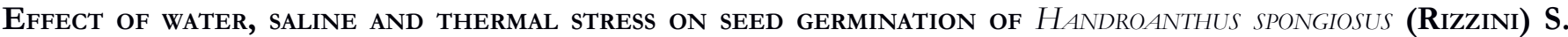
Grose (Bignoniaceae)

ABstract - The aim of this study was to evaluate fruit morphometry and germinative responses of Handroanthus spongiosus (Rizzini) S. Grose (Bignoniaceae) seeds submitted to environmental factors, as well as to contribute to the knowledge of the ecophysiology of this species threatened with extinction. Seeds were collected in the municipality of Casa Nova, located north of the State of Bahia. It was determined the ideal temperature for seed germination, besides evaluating the effect of temperature and water and saline stress on seed germination. The weight, length and quantity of seeds in each fruit of the species were also evaluated. It was observed that there is no uniformity in the size and quantity of seeds present in the fruits. The ideal temperature for seed germination was $25^{\circ} \mathrm{C}$ and the species showed tolerance to a temperature range of 15 to $35^{\circ} \mathrm{C}$. It was also observed that $H$. spongiosus was not tolerant to water and saline stress, which may be related to the restricted distribution of the species in some Caatinga regions.

Keywords: CAATINGA; CASCUdo; W ATER AVAILABILITY; SALINITY; TeMPERATURE.

EFECTO DE LOS ESTRESES HÍDRICO, SALINO Y TÉRMICO EN LA GERMINACIÓN DE LAS SEMILLAS DE HANDROANTHUS ESPONGIOSUS (Rizzini) S. Grose (Bignoniaceae) 
Resumen - Se objetivó evaluar la morfometría del fruto y las respuestas germinativas de las semillas de Handroanthus espongiosus (Rizzini) S. Grose (Bignoniaceae) sometidas a factores ambientales, así como contribuir al conocimiento de la ecofisiología de esa especie amenazada de extinción. Se recolectaron las semillas en el municipio de Casa Nova, localizado en la región Norte del Estado de Bahía. Se determinó la temperatura ideal para la germinación de las semillas, además de evaluar el efecto de la temperatura y de los estreses hídrico y salino en la germinación de las semillas. También se evaluaron el peso, la longitud y la cantidad de semillas en cada fruto de la especie. Se observó que no hay una uniformidad en el tamaño y la cantidad de semillas presentes en los frutos. La temperatura ideal para la germinación de las semillas fue $25^{\circ} \mathrm{C}$ y la especie se mostró tolerante a un rango de temperatura de 15 a $35^{\circ} \mathrm{C}$. Se observó, además, que H. espongiosus no se mostró tolerante a los estreses hídrico y salino, lo que puede estar relacionado a la distribución restringida de la especie en algunas regiones de la Caatinga.

Palabras clave: CaAtinga; Cascudo; Disponibilidad Hídrica; Salinidad; Temperatura.

\section{INTRODUÇÃO}

Os estudos morfológicos auxiliam na interpretação dos testes de germinação em laboratório, orientam quanto ao armazenamento de sementes e métodos de cultivo, além de facilitarem o reconhecimento das espécies em banco de sementes no solo (Donadio e Demattê 2000, Larcher 2000). Da mesma forma, estudos que avaliem como o estresse influencia o processo de germinação de sementes são de fundamental importância na ecofisiologia das sementes, no sentido de avaliar os limites de tolerância e a capacidade de adaptação das espécies (Donadio e Demattê 2000, Larcher 2000).

Entender como as espécies ameaçadas de extinção podem responder aos diversos fatores ambientais representa uma alternativa ao manejo dessas espécies. A capacidade das sementes germinarem sob diferentes fatores abióticos é de fundamental importância para sobrevivência das espécies vegetais (Ceccon et al. 2006). Fatores abióticos como a luz, a temperatura, a disponibilidade de água e a salinidade são fundamentais na germinação e no desenvolvimento inicial de plantas que habitam ambientes semiáridos como a Caatinga (Kigel 1995).

Alguns trabalhos foram realizados avaliando-se os estresses hídrico e salino, além de diferentes temperaturas. Entre esses estudos, merecem destaque o de Meiado et al. (2010) com Cereus jamacaru DC. subsp. jamacaru (Cactaceae), Lima e Torres (2009) com Ziziphus joazeiro Mart. e ainda trabalhos com espécies da família Bignoniaceae, como o de Cabral et al. (2003) com Tabebuia aurea (Silva Manso) Benth. \& Hook.f. ex S. Moore e o de Socolowski e Takaki (2004) com Jacaranda mimosifolia (D. Don). Apesar desses e de vários outros estudos, pouco se sabe sobre as respostas germinativas de espécies endêmicas e ameaçadas de extinção, como Handroanthus spongiosus (Rizzini) S. Grose (Bignoniaceae) e esses estudos são fundamentais para entender a capacidade fisiológica das plantas de se adaptarem a ambientes semiáridos como a Caatinga.

Popularmente conhecida como cascudo, H. spongiosus é uma espécie arbórea que atinge até oito metros de altura, com dispersão anemocórica e ocorrência preferencialmente em solos arenosos da Caatinga (Espírito Santo 2012). Essa espécie encontra-se classificada na categoria de ameaça "em perigo de extinção" (EN), segundo a lista oficial de espécies da flora ameaçadas de extinção (Brasil 2008). O objetivo deste estudo foi avaliar a morfometria do fruto e as respostas germinativas de $H$. spongiosus a alguns fatores ambientais, assim como contribuir para o conhecimento da ecofisiologia da espécie. 


\section{Material e Métodos}

\section{Local de coleta dos frutos}

Para a análise das respostas germinativas de H. spongiosus aos fatores abióticos de temperatura, salinidade e estresse hídrico, foram coletados frutos em cerca de 20 árvores da espécie estudada, no Sítio Morrinho, município de Casa Nova, região Norte do Estado da Bahia (09¹6'20,55’W; 41¹5'03,02”S), no mês de novembro de 2012. A região é caracterizada pelo clima Semiárido (BShW), com precipitação média atual de $409 \mathrm{~mm}$ e solos do tipo Neossolo Quartzarênico Órtico (Brasil 2006), sendo considerada uma área prioritária para a conservação da Caatinga, classificada como "Insuficientemente Conhecida" (Brasil 2007).

\section{Descrição morfológica dos frutos}

Para a descrição dos frutos quanto ao seu tamanho, quantidade de sementes e peso dos frutos com e sem sementes foram selecionados 30 frutos, escolhidos ao acaso para medição individual. Os frutos foram pesados em balança analítica com precisão de $0,001 \mathrm{~g}$ e mensurados quanto ao comprimento utilizando-se régua. $\mathrm{O}$ comprimento foi medido da base até o ápice. Os dados das características quantitativas foram submetidos à análise descritiva, onde se calculou a média aritmética e desvio padrão.

\section{Tratamentos de temperatura}

Inicialmente, para se verificar a temperatura ideal para a germinação de sementes de $H$. spongiosus, foram avaliados sete tratamentos de temperaturas constantes: 15, 20, 25, 30, 35, 40 e $45^{\circ} \mathrm{C}$. Em cada tratamento foram colocadas 100 sementes para germinar em placas de Petri forradas com dupla camada de papel filtro umedecido com água destilada, sendo mantidas em câmara de germinação, sob luz branca e fotoperíodo de 12 horas. A germinação foi avaliada diariamente, por um período de 30 dias, e o critério para se considerar sementes germinada foi a protrusão radicular de tamanho $\geq 2 \mathrm{~mm}$ (Meiado et al. 2013).

\section{Tratamento de estresse hidrico e estresse salino}

Para os tratamentos de estresses hídrico e salino foram utilizados soluções comerciais de polietileno glicol (PEG 6000) (Villela et al. 1991) e cloreto de sódio (Braccini et al. 1996) para simular o estresse hídrico e o estresse salino, respectivamente. Em ambos os experimentos foram avaliados os potenciais osmóticos de 0,0 (água destilada); -0,2; -0,4; -0,6; -0,8 e -1,0 MPa, e as placas de Petri foram mantidas em temperatura de $25^{\circ} \mathrm{C}$, sob luz branca e fotoperíodo de 12 horas. O potencial osmótico foi calculado de acordo com Villela et al. (1991) e Braccini et al. (1996). Nenhuma solução foi adicionada às placas durante o experimento. A germinação foi avaliada diariamente, por um período de 30 dias. 
Ao final das análises foram calculados a germinabilidade (\%), o tempo médio de germinação $\left[\mathrm{t}=\sum\right.$ ni. ti/ $\sum$ ni, onde ti é o período desde o início do experimento até a enésimas observação (dias) e ni é o número de sementes germinadas no tempo i] e o índice de sincronização [E $=-\sum$ fi.log2 fi, onde fi é a frequência relativa da germinação (i.e., a proporção de sementes germinadas em um intervalo)] de acordo com Ranal e Santana (2006).

Todos os parâmetros de germinação foram comparados para sua significância utilizando um teste ANOVA seguidos pelo teste de Tukey $(\phi \leq 0,05)$, sendo os dados expressos em valores médios e desvio padrão. A normalidade dos dados e a homogeneidade das variâncias foram verificadas através dos testes Levene e Shapiro Wilk, respectivamente (Zar 1999). Todas as análises estatísticas foram realizadas no software SISVAR 5.1C (Ferreira 2007).

\section{Resultados e Discussão}

Os frutos de H. spongiosus não apresentaram uniformidade quanto ao comprimento e ao número de sementes presentes em cada fruto. O comprimento do fruto variou entre 8,1 a 19,8 cm, o número de sementes entre 97 e 192 sementes, o peso dos frutos com sementes entre 0,89 e 2,17 g e o peso dos frutos sem sementes entre 0,41 e $0,97 \mathrm{~g}$. Os seguintes valores médios foram observados para o comprimento do fruto $(14,33 \pm 2,82$ $\mathrm{cm})$, o número de sementes $(127,5 \pm 32,97$ sementes), o peso do fruto com sementes $(1,20 \pm 616,2 \mathrm{~g})$ e o peso do fruto sem sementes $(0,60 \pm 0,16 \mathrm{~g})$.

As diferenças quanto ao tamanho dos frutos podem estar relacionadas à diversidade genética da espécie na população. Acredita-se, ainda, que a modificação em tamanho de frutos não esteja relacionada apenas a diversidade genética, mas também às condições determinadas pelo meio ambiente, como o estresse hídrico ocasionado pela seca na Caatinga, o que desencadeiam alterações funcionais (Oliveira et al. 2010).

O presente estudo mostrou diferenças significativas na germiabilidade de sementes de $H$. spongiosus em relação às diferentes temperaturas avaliadas ( $\mathrm{F}=34,92 ; \mathrm{gl}=6 ; \mathrm{p}=<0,0001$; Figura 1).

Figura 1 - Germinabilidade (\%) de sementes de Handroanthus spongiosus (Rizzini) S. Grose (Bignoniaceae) submetidas a diferentes tratamentos de temperaturas constantes $\left({ }^{\circ} \mathrm{C}\right)$.

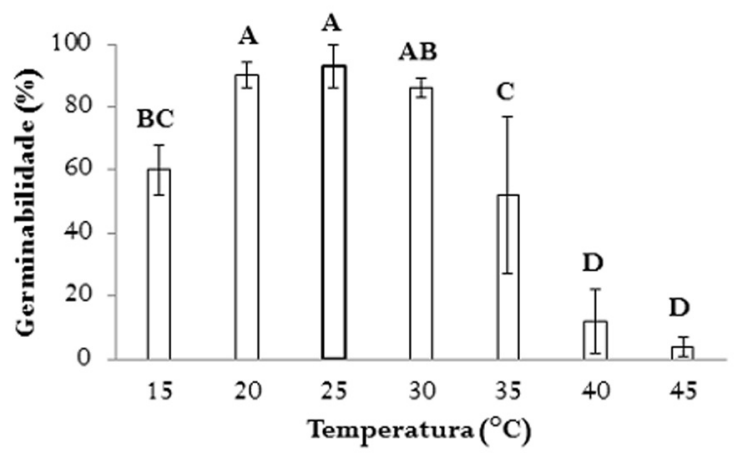

Observou-se, ainda, que a temperatura influenciou o tempo médio de germinação $(\mathrm{F}=16,84$; $\mathrm{gl}=6$; $\mathrm{p}<$ 0,0001) e o índice de sincronização $(F=10,39 ; \mathrm{gl}=6 ; \mathrm{p}<0,0001)$ (Tabela 1). Os resultados mostraram ainda que apesar de as temperaturas de 20 e $25^{\circ} \mathrm{C}$ não diferirem significativamente quanto à germiabilidade e ao tempo médio de germinação, a temperatura de $25^{\circ} \mathrm{C}$ foi escolhida como a temperatura ideal para a germinação de espécies por apresentar-se com os melhores valores entre todos os parâmetros avaliados, assim como também foi observado em estudo feito por Socolowski e Takaki (2004) com Jacaranda mimosifolia (D. Don) (Bignoniaceae), 
onde a temperatura de $25^{\circ} \mathrm{C}$ também foi considerada como a ideal. Em relação à faixa de temperatura favorável à germinação, apesar de não apresentar uma uniformidade quanto aos parâmetros de tempo médio de germinação e índice de sincronização, a germiabilidade se mostrou favorável nas faixas de 15 a $35^{\circ} \mathrm{C}$, bem como observado para a espécie T. aurea, como destaca Cabral et al. (2003).

Tabela 1 - Tempo médio de germinação (dias) e índice de sincronização da germinação de sementes de Handroanthus spongiosus (Rizzini) S. Grose (Bignoniaceae) submetidas a diferentes tratamentos de temperaturas constantes $\left({ }^{\circ} \mathrm{C}\right)$. Dados expressos em média \pm desvio padrão. Letras minúsculas indicam diferenças significativas entre os tratamentos $(\mathrm{p}<$ $0,05)$.

\begin{tabular}{ccc}
\hline Temperatura $\left({ }^{\circ} \mathbf{C}\right)$ & $\begin{array}{c}\text { Tempo Médio de Germinação } \\
\text { (dias) }\end{array}$ & Índice de Sincronização \\
\hline $\mathbf{1 5}$ & $6,770 \pm 0,609 \mathrm{c}$ & $1,638 \pm 0,189 \mathrm{a}$ \\
$\mathbf{2 0}$ & $2,503 \pm 0,080 \mathrm{a}$ & $1,251 \pm 0,085 \mathrm{ab}$ \\
$\mathbf{2 5}$ & $2,116 \pm 0,134 \mathrm{a}$ & $0,226 \pm 0,264 \mathrm{c}$ \\
$\mathbf{3 0}$ & $2,000 \pm 0,078 \mathrm{a}$ & $0,625 \pm 0,666 \mathrm{bc}$ \\
$\mathbf{3 5}$ & $3,820 \pm 0,352 \mathrm{ab}$ & $1,023 \pm 0,200 \mathrm{abc}$ \\
$\mathbf{4 0}$ & $4,679 \pm 0,472 \mathrm{~b}$ & $0,787 \pm 0,529 \mathrm{bc}$ \\
$\mathbf{4 5}$ & $2,500 \pm 2,082 \mathrm{a}$ & - \\
\hline
\end{tabular}

- germinabilidade nula.

Em relação aos resultados quando as sementes foram submetidas a diferentes concentrações de solução de PEG 600 foi observado que a germinabilidade diminuiu à medida que se diminuiu a disponibilidade de água (F $=54,698 ; \mathrm{gl}=5 ; \mathrm{p}<0,0001 ;$ Figura 2), onde também foram afetados o tempo médio de germinação $(\mathrm{F}=3,097$; $\mathrm{gl}=5 ; \mathrm{p}<0,003$; Tabela 2) e índice de sincronização $(\mathrm{F}=6,466 ; \mathrm{gl}=5 ; \mathrm{p}<0,0001$; Tabela 02). Foi possível observar com estes resultados que há redução significativa na germinação de sementes de $H$. spongiosus onde haja uma baixa disponibilidade de água. Resultados semelhantes foram observados em estudos realizados com outras espécies da Caatinga como em estudo realizado por Meiado et al. 2010 com C. jamacaru subsp. jamacaru e Lima e Torres 2009 com Z. joazeiro. Essas espécies também demonstraram uma baixa porcentagem de germinação à medida que se diminuía a disponibilidade de água (Lima e Torres 2009, Meiado et al. 2010).

Tabela 2 - Tempo médio de germinação (dias) e índice de sincronização da germinação de sementes de Handroanthus spongiosus (Rizzini) S. Grose (Bignoniaceae) submetidas estresse hídrico simulado com soluções de polietileno glicol 6000 (PEG 6000) com diferentes potenciais osmóticos (MPa). Dados expressos em média \pm desvio padrão. Letras minúsculas indicam diferenças significativas entre os tratamentos $(p<0,05)$.

\begin{tabular}{ccc}
\hline Solução PEG 6000 (MPa) & Tempo Médio de Germinação (dias) & Índice de Sincronização \\
\hline $\mathbf{0 , 0}$ & $3,778 \pm 1,454 \mathrm{a}$ & $1,540 \pm 0,274 \mathrm{a}$ \\
$\mathbf{- 0 , 2}$ & $3,605 \pm 0,965 \mathrm{a}$ & $1,444 \pm 0,264 \mathrm{a}$ \\
$\mathbf{- 0 , 4}$ & $3,185 \pm 1,020 \mathrm{a}$ & $1,283 \pm 0,553 \mathrm{a}$ \\
$\mathbf{- 0 , 6}$ & $3,242 \pm 0,594 \mathrm{a}$ & $1,024 \pm 0,427 \mathrm{a}$ \\
$\mathbf{- 0 , 8}$ & $4,250 \pm 3,686 \mathrm{~b}$ & $0,964 \pm 0,725 \mathrm{~b}$ \\
$\mathbf{- 1 , 0}$ & - & - \\
\hline
\end{tabular}

- germinabilidade nula. 
Figura 2 - Germinabilidade (\%) de sementes de Handroanthus spongiosus (Rizzini) S. Grose (Bignoniaceae) submetidas estresse hídrico simulado com soluções de polietileno glicol 6000 (PEG 6000) com diferentes potenciais osmóticos (MPa).

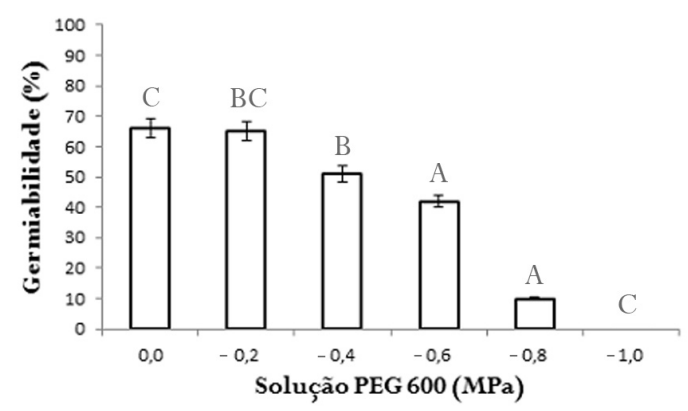

Quando foi observada a germinação das sementes em altas concentrações salinas também foi observada a diminuição da germinabilidade de H. spongiosus ( $\mathrm{F}=74,01$; $\mathrm{gl}=5$; $\mathrm{p}<0,0001$; Figura 3).

Figura 3. Germinabilidade (\%) de sementes de Handroanthus spongiosus (Rizzini) S. Grose (Bignoniaceae) submetidas estresse salino simulado com soluções de cloreto de sódio PA (NaCl) com diferentes potenciais osmóticos (MPa).

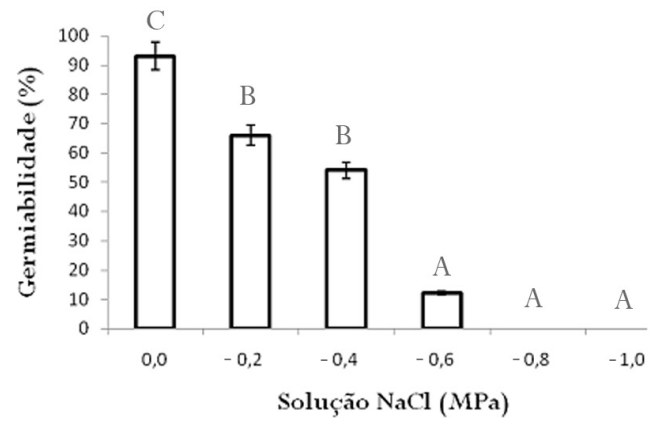

Foi possível observar que nas concentrações de -0,6; -0,8 e -1,0 $\mathrm{MPa}$ de $\mathrm{NaCl}$ não houve germinação de sementes, assim como essas concentrações também influenciaram o tempo médio de germinação ( $F=130,20$; $\mathrm{gl}=5 ; \mathrm{p}<0,0001$; Tabela 3) e o índice de sincronização $(\mathrm{F}=24,598 ; \mathrm{gl}=5 ; \mathrm{p}<0,0001$; Tabela 3). A bibliografia já relata a influência significativa da condição de salinidade dos solos em relação a germinação de sementes. $O$ alto teor de sais, especialmente de cloreto de sódio $(\mathrm{NaCl})$, pode inibir a germinação devido a diminuição do potencial osmótico, ocasionando prejuízos as demais fases do processo (Lima et al. 2005). Assim como no presente estudo, a influência negativa da salinidade na germinação de sementes também pode ser observada em estudo feito com a espécie Mimosa tenuiflora (Willd.) Poir., onde as porcentagens finais de germinação diminuíram a medida em que se aumentava as concentrações de $\mathrm{NaCl}$ da solução (Bakke et al. 2006).

Tabela 3 - Tempo médio de germinação (dias) e índice de sincronização da germinação de sementes de Handroanthus spongiosus (Rizzini) S. Grose (Bignoniaceae) submetidas estresse salino simulado com soluções de cloreto de sódio PA $(\mathrm{NaCl})$ com diferentes potenciais osmóticos $(\mathrm{MPa})$. Dados expressos em média \pm desvio padrão. Letras minúsculas indicam diferenças significativas entre os tratamentos $(\mathrm{p}<0,05)$.

\begin{tabular}{ccc}
\hline Solução NaCl (MPa) & Tempo médio de Germinação (dias) & Índice de Sincronização \\
\hline $\mathbf{0 , 0}$ & $2,115 \pm 0,134 \mathrm{a}$ & $0,138 \pm 0,189 \mathrm{~b}$ \\
$\mathbf{- 0 , 2}$ & $2,453 \pm 0,313 \mathrm{ab}$ & $0,901 \pm 0,302 \mathrm{a}$ \\
$\mathbf{- 0 , 4}$ & $2,954 \pm 0,430 \mathrm{~b}$ & $1,287 \pm 0,271 \mathrm{a}$ \\
$\mathbf{- 0 , 6}$ & $4,693 \pm 0,549 \mathrm{c}$ & $1,377 \pm 0,456 \mathrm{a}$ \\
$\mathbf{- 0 , 8}$ & - & - \\
$\mathbf{- 1 , 0}$ & - & - \\
\hline
\end{tabular}

- germinabilidade nula. 
Os fatores abióticos exercem um importante papel no controle de eventos germinativos das espécies na Caatinga, merecendo destaque a disponibilidade hídrica do solo. Informações sobre respostas germinativas de sementes submetidas a diferentes fatores abióticos, como a disponibilidade de água do solo, a temperatura e a salinidade nos solos, podem ser úteis para a compreensão dos padrões de distribuição geográfica das espécies em escala de populações e comunidades naturais, principalmente em estudos para o manejo de espécies ameaçadas de extinção como H. spongiosus (Barbosa 2003).

\section{Conclusão}

O cascudo, como é popularmente conhecida a espécie H. spongiosus, não se mostrou tolerante a fatores como o estresse hídrico e o estresse salino, apesar de apresentar uma ampla faixa de tolerância em relação às diferentes temperaturas. Além disso, os frutos da espécie apresentaram grande variedade em relação ao peso, comprimento e quantidade de sementes por fruto, o que demonstra uma elevada variedade genética dentro da população estudada.

\section{REFERÊNCIAS}

Bakke IA, Freire ALO, Bakke OA, Andrade AP e Bruno RLA. 2006. Water and Sodium Chloride Effects on Mimosa tenuiflora (Willd.) Poiret Seed Germination. Revista Caatinga, 19(3): 261-267.

Barbosa DCA. 2003. Estratégias de germinação e crescimento de espécies lenhosas da Caatinga com germinação rápida. In: Leal IR, Tabarelli M e Silva JMC (Eds.). Ecologia e conservação da Caatinga. Recife: Editora Universitária da UFPE, p. 625-656.

Braccini AL, Ruiz HA, Braccini MCL e Reis MS. 1996. Germinação e vigor de sementes de soja sob estresse hídrico induzido por soluções de cloreto de sódio, manitol e polietileno glicol. Revista Brazileira de Sementes, 18(1): 10-16.

Brasil - Ministério do Planejamento, Orçamento e Gestão, Instituto Brasileiro de Geografia e Estatística. 2006. Sistema Brasileiro de Classificação de Solos. Rio de Janeiro: Instituto Brasileiro de Geografia e Estatística.

Brasil - Ministério do Meio Ambiente. 2007. Áreas Prioritárias para Conservação da Biodiversidade Bioma Caatinga. Brasília: Ministério do Meio Ambiente.

Brasil - Ministério do Meio Ambiente. 2008. Instrução Normativa n 6, 23 de setembro de 2008. Brasília: Ministério do Meio Ambiente.

Cabral EL, Barbosa DCA e Simabukuro EA. 2003. Armazenamento e germinação de sementes de Tabebuia aurea (Manso) Benth \& Hook.F. ex. S. Moore. Acta Botanica Brasilica, 17(4): 609-617.

Ceccon E, Huante P e Rincón E. 2006. Abiotic Factors Influencing Tropical Dry Forests Regeneration. Brazilian Archives of Biology and Technology, 49(2): 305-312. 
Donadio NMM e Demattê MESP. 2000. Morfologia de frutos, sementes e plântulas de canafístula (Peltophorum dubium (Spreng) Taub.) e jacarandá-da-Bahia (Dalbergia nigra (Vell.) Fr. All. ex Benth.) - Fabaceae. Revista Brasileira de Sementes, 22(1): 64-73.

Espírito Santo FS. 2012. A aliança Tabebuia (Bignoniaceae) no Estado da Bahia, Brasil. Dissertação de Mestrado. Feira de Santana: Universidade Estadual de Feira de Santana.

Ferreira DF. 2007. Sisvar versão 5.1 (Build 72). DEX/UFLA, Minas Gerais, Lavras.

Kigel J. 1995. Seed Germination in Arid and Semiarid Regions. In: Kigel J e Galili G. (Eds). Seed Development and Germination. New York: Marcel Dekker, p. 645-699.

Larcher W. 2000. Ecofisiologia vegetal. São Carlos: Rima, 531p.

Lima MGS, Lopes NF, Moraes DM e Abreu CM. 2005. Qualidade fisiológica de sementes de arroz submetidas a estresse salino. Revista Brasileira de Sementes, 27(1): 54-61.

Lima BG e Torres SB. 2009. Estresse hídrico e salino na germinação de sementes de Zizyyphus joaz̧eiro Mart. (Rhamnaceae). Revista Caatinga, 22(4): 93-99.

Meiado MV, Albuquerque LSC, Rocha EA, Rojas-Aréchiga M e Leal IR. 2010. Seed Germination Responses of Cereus jamacaru DC. ssp. jamacaru (Cactaceae) to Environmental Factors. Plant Species Biology, 25(2): 120-128.

Meiado MV, Simabukuro EA e Iannuzzi L. 2013. Entomofauna Associated to Fruits and Seeds of Two Species of Enterolobium Mart. (Leguminosae): Harm or Benefit? Revista Brasileira de Entomologia, 57(1): 100-104.

Nogueira FCB, Medeiros Filho S e Gallão MI. 2010. Caracterização da germinação e morfologia de frutos, sementes e plântulas de Dalbergia cearensis Ducke (pau-violeta) - Fabaceae. Acta Botanica Brasilica, 24(4): 978985.

Ranal MA e Santana DG. 2006. How and Why to Measure the Germination Process? Revista Brasileira de Botânica, 29(1): 1-11.

Socolowski F e Takaki M. 2004. Germination of Jacaranda mimosifolia (D. Don - Bignoniaceae) Seeds: Effects of Light, Temperature and Water Stress. Brazilian Archives of Biology and Technology, 47(5): 785-792.

Villela FA, Doni Filho L e Sequeira EL. 1991. Tabela de potencial osmótico em função da concentração de Polietileno Glicol 6.000 e da temperatura. Pesquisa Agropecuária Brasileira, 26(11/12): 1957-1968.

Zar JH. 1999. Biostatistical Analysis. New Jersey: Prentice-Hall Inc., 663p. 